\title{
Analysis of wave damping in pipeline having different pipe materials configuration under water hammer conditions
}

Rahul Kumar Garg ( $\sim$ rahulgargmech@gmail.com )

IIT Roorkee: Indian Institute of Technology Roorkee https://orcid.org/0000-0001-6119-7749

Arun Kumar

IIT Roorkee: Indian Institute of Technology Roorkee

Ali Abbas

Madanapalle Institute of Technology and Science

\section{Research Article}

Keywords: water hammer, wave damping coefficient, water conductor system, method of characteristic (MOC), MS and GRP pipelines

Posted Date: March 30th, 2021

DOl: https://doi.org/10.21203/rs.3.rs-178392/v1

License: (a) (1) This work is licensed under a Creative Commons Attribution 4.0 International License. Read Full License 


\title{
Analysis of wave damping in pipeline having different pipe materials configuration under water hammer conditions
}

\author{
Rahul Kumar Garg ${ }^{1}$, Arun Kumar ${ }^{2}$ and Ali Abbas ${ }^{3}$ \\ ${ }^{1,2}$ Department of Hydro and Renewable Energy, Indian Institute of Technology Roorkee, \\ Roorkee - 247667, Uttarakhand, India \\ ${ }^{3}$ Madanapalle Institute of Technology \& Science, Madanapalle, A.P. \\ ${ }^{1}$ Corresponding Author: E- mail: rahulgargmech@gmail.com,
}

\begin{abstract}
Hydraulic transient occurs whenever there is a sudden change in the flow velocity resulting in variation of pressure and flow in a water conductor system. Experiments have been conducted in a straight pipeline having material of Mild Steel (MS) and Glass Fibre Reinforced Plastic (GRP) pipelines and their combined configurations. From experiments, it has been observed that there is a smooth and strong damping of pressure waves in the pipeline system. Experimental results were compared with results obtained for classical water hammer equations solved in MATLAB and analyzed that there are several dissipative factors, other than fluid viscosity, responsible for strong damping of pressure wave amplitude. Further, an improvement in the governing equation of water hammer in a closed conduit was proposed by incorporating a different wave damping coefficient $(\alpha)$. The modified governing equations have been solved for each water hammer cycle using MATLAB. The numerical simulation results show that proposed approach gives better agreements between the experimental and computational results for all investigated cases.
\end{abstract}


Keywords: water hammer, wave damping coefficient, water conductor system, method of characteristic (MOC), MS and GRP pipelines

\section{Introduction}

Hydraulic transient or water hammer occur whenever there is a sudden alteration in the flow velocity resulting in variation of pressure and discharge in a water conductor system for hydropower and water supply plants $[1,2]$. It is very important to keep hydraulic transient with in safe limits in a water conductor system for a viewpoint of hydropower system safety, effective operation and to increase its life span. Uncontrolled transient events may cause serious impact on both civil and mechanical infrastructure of the plants [3-5]. It is difficult for power and water utilities to reduce water hammer formation in water conductor system due to the variation in demand. The analysis of hydraulic transient is essential for the selection of penstock material, its pressure class and the specifications of surge protection devices. Formation of hydraulic transient in penstock is greatly influenced by its material $[6,7]$. More rigid material means higher transient formation and elastic materials forms lower transient waves. The viscoelastic pipes suppress water hammer pressure effectively because of its low characteristics impedance and fast damping compared with metallic pipes $[8,9]$.

The general simplified continuity and momentum equations used for transient flow analysis in a closed conduits consist of two hyperbolic partial differential equations shown as Eq. (1) and (2) respectively $[10,11]$ which does not provide direct solution by any numerical method for given boundary conditions. The water hammer wave velocity (celerity) for an elastic pipeline with circular cross section can be estimated with Eq. (3) [12,13].

$$
\frac{\partial H}{\partial t}+\frac{a^{2}}{g A} \frac{\partial Q}{\partial x}=0
$$




$$
\begin{aligned}
& \frac{\partial Q}{\partial t}+g A \frac{\partial H}{\partial x}+R Q|Q|=0 \\
& a=\sqrt{\frac{1}{\rho\left(\frac{1}{K}+\frac{D}{E \cdot T}\right)}}
\end{aligned}
$$

where, $A=$ cross section flow area, $g=$ gravitational constant, $R=$ pipeline resistance coefficient, $a=$ wave velocity (celerity), $H(x, t)=$ pressure head; and $Q(x, t)=$ flow through the pipeline. $x=$ distance along the pipeline; $t=$ time, $\rho=$ fluid density, $K=$ bulk modulus of fluid, $D=$ pipeline inside diameter, $E=$ young modulus of pipe wall material and $T=$ pipeline thickness.

Researchers across the globe have studied the water hammer impact on penstock material experimentally or analytically. Mitosek et al. [14] conducted experiments for water hammer analysis in two different material pipelines and proposed some modification in governing equation of the water hammer for smooth damping of pressure waves. Adamkowski et al. [15] conducted the experiments to study the damping effect of the pressure waves in the pipeline and concluded that the classical water hammer theory cannot predict the damping effect properly. Gong et al. [8] proposed a surge suppression technique by using metallic-viscoelastic-metallic configuration in water distribution system. Larson et al. [9] studied transient effect in water and sewage pipes by measuring pressure and strain in pipes made by different materials and concluded that the response of pipes during transient events can be analyzed by using linear elastic theory with related modulus of the elasticity. Duan et al. [16] highlighted the effect of unsteady friction in viscoelastic pipes by implementing quasi $2 \mathrm{D}$ numerical method and concluded that the water hammer equations were not capable to exhibits the damping behavior during water hammer events. 
Several researchers had studied the effect of the shear stress in wave damping and smoothing of the unsteady flow $[14,17,18]$. It was further identified that apart from shear stress, there are some other factors which influences the damping and smoothing of pressure waves in the water hammer phenomenon. The friction force only influences the amplitude of celerity, whereas, there are some dissipative effect which affect the wave damping. There are various sources of energy dissipation in water hammer events. In this paper, the effect of material elasticity, one of dissipative effect is discussed and its effect is incorporated in the water hammer equations. This source is related with both fluid and pipe wall material elasticity which can be taken into account by introducing variable wave pressure velocity in the continuity equation of water hammer. The water hammer experiments were carried out for metallic (MS) and viscoelastic (GRP) pipeline and results obtained using improved MOC codes were validated.

\section{Experimental Set up}

The experimental set up for hydraulic transient test was designed and fabricated in the Department of Hydro and Renewable Energy at Indian Institute of Technology Roorkee. The experimental set up consist of two straight pipelines with different materials, a butterfly valve (7) mounted on the downside of the pipeline, an air vessel (5) and pressure sensors (10). The water hammer experiments were carried out for metallic (MS) and viscoelastic (GRP) pipeline. The detail characteristics of the pipe materials are given in Table 1.

The pipes were fixed to the floor with supports. The water supply was given to the experimental setup from the reservoir (8) by a centrifugal pump (1). The butterfly valve (7) was operated by pneumatic actuator. The valve closure time $t_{C}$, was calculated as $0.06 \mathrm{~s}$. The pressure was measured with the help of five pressure sensors connected with a DAQ system (10), located 
at equi-distance on the test section of the pipe. The schematic diagram of water hammer experimental setup shown in Fig. 1.

The flow rate, $Q_{0}$, was measured with a ultrasonic flow meter with an accuracy of $0.5 \%$ and controlled by gate valves located at various locations on the experimental set up, The initial tests were conducted for three different velocities and data acquired from sensors have been analyzed. The water hammer effect was created in the test section with the help of fast closing butterfly valve. A gate valve, installed at downstream of the butterfly valve is used to maintain the required head and discharge in the test section. The different pipe line material and their combined configuration in the experimental setup are shown as Fig. 2

\section{MATHMATICAL FORMULATION OF ELASTIC ENERGY EFFECT IN MODIFIED WATER HAMMER EQUATION}

The water hammer events can be considered as a 1D phenomenon in which both the bulk modulus of elasticity and fluid density change less within a large range of pressure values $[19,20]$. The celerity or pressure wave velocity is usually considered to be constant throughout in the numerical simulation. As per the result which is obtained from Eq. (3), for a given pipeline diameter and fluid the celerity remains unchanged. However, the time period parameters $\left(T_{i}, T_{i i}\right.$, $\mathrm{T}_{\mathrm{iii}}$ and $\mathrm{T}_{\mathrm{iv}}$ ) can alter the celerity (Fig. 3) where function $H(t)$ is asymmetrical. In each step of $H(t)$, there are four segments having specific curvatures. This variation can be related with the pressure wave phase. There are four time intervals with different lengths in one wave cycle. The Fig. 3 shows the enlarged view of graph of transient head oscillation in one water hammer cycle in MS pipeline. 
It can be analyzed and observed that the time of pressure increase is shorter than the time of pressure decrease. This difference is related with the pressure wave amplitude, because as the amplitude decreases, it disappears with time. As these time intervals have different lengths, their sum, being the total wave period is constant with time. The asymmetry observed in $H(t)$ must be related with the celerity. Therefore, instead of $a=$ constant, $a=a(x, t)$ should be assumed in simulation process $[14,21]$. By taking the accounts of varying gradients of function $H(x, t)$, in phase of compression, when the fluid flow slow down, the wave travels at a speed less than constant celerity $[a(x, t)<a($ cont.$)]$, otherwise in the phase of decompression, when the flow accelerates, the wave travels at a speed greater than the average $[a(x, t)>a($ cont. $)]$. During the compression phase of the fluid in the pipeline, a fraction of energy, denoted as $E_{\text {diss }}$, is lost because of dissipation. The principal of energy conservation can also be written as Eq. (4) [22].

$E_{\text {kin }}=E_{f}+E_{m}+E_{\text {diss }}$

During compression wave damping coefficient $\left(\alpha_{1}\right)$ may be defined as per Eq. (5)

$$
\alpha_{1}=\frac{E_{\text {diss }}}{E_{f}+E_{m}}
$$

where

$E_{k i n}=\rho \cdot g \cdot A_{p} \cdot \Delta x \frac{V_{0}}{2 g}, \quad E_{f}=\Delta p^{2} \frac{A_{p} \cdot \Delta x}{2 K}, \quad E_{m}=\Delta p^{2} \cdot A_{p} \cdot \frac{D \cdot \Delta x}{2 \cdot E \cdot e}, \quad \Delta p=\rho \cdot g \cdot \Delta H$

and $A_{p}=$ cross section area of the pipeline, $\Delta x=$ pipeline sections, $\Delta p=$ pressure increment and $\Delta H=$ increment in pressure head.

The modified pressure increment $\Delta p$ during compression may be expressed as per Eq. (6) 
$\Delta p=V_{0} \cdot \rho \cdot \frac{\sqrt{\frac{K}{\rho}}}{\sqrt{1+\alpha_{1}} \sqrt{1+\frac{K \cdot D}{E \cdot e}}}$

In the phase of decompression, where an increment in the flow velocity occurs $\left(d V_{0} / d t>0\right)$, the store energy due to the elasticity and pipe material is returned as the kinetic energy of the following water. However, due to dissipation, only small fraction of the stored energy is return back. Thus, the energy balance and change in pressure due to water hammer events can be expressed as Eqs. (7) and (8) respectively.

$E_{k i n}=E_{f}+E_{m}-E_{\text {diss }}$

$\Delta p=V_{0} \cdot \rho \cdot \frac{\sqrt{\frac{K}{\rho}}}{\sqrt{1-\alpha_{2}} \sqrt{1+\frac{K \cdot D}{E \cdot e}}}$

where, $a_{d}=\frac{a}{\sqrt{1-\alpha_{2}}}, \alpha_{2}=$ wave damping coefficient during decompression phase.

The relationship between pressure wave velocities $a_{c}$ and $a_{d}$ defines the standard wave velocity as Eq. (9).

$$
a=2 \cdot \frac{a_{c} \cdot a_{d}}{a_{c}+a_{d}}
$$

The Eq. (9) provides the standard wave velocity $a=$ constant in the water hammer pressure phenomenon.

For small values of $\alpha_{1}$ and $\alpha_{2}\left(\alpha_{1}, \alpha_{2}<0.1\right)$, it can be assumed that $\alpha_{1} \cong \alpha_{2}=\alpha$. If $\alpha_{1}<0.1$ it implies that the value of $\alpha_{2}$ satisfying the relation in Eq. (8) must differ from the value of $\alpha_{1}$ by 
less than $1 \%$. The important point in the proposed approach is to develop a suitable formula for the space and time variation of the pressure waves. To modify the pressure wave celerity, the correction process is carried out in the following steps [14]:

Step 1: if $H(x, t)>H_{0}$ and $\frac{\partial H(x, t)}{\partial t}<0$

$$
\text { then } \left.a(x, t)=\frac{a}{\sqrt{1-\alpha_{2}}} \quad \text { (for segment } T_{i}\right)
$$

Step 2: if $H(x, t)<H_{0}$ and $\frac{\partial H(x, t)}{\partial t}<0$

$$
\text { then } \left.a(x, t)=\frac{a}{\sqrt{1+\alpha_{1}}} \text { (for segment } T_{i i}\right)
$$

Step 3: if $H(x, t)<H_{0}$ and $\frac{\partial H(x, t)}{\partial t}>0$

$$
\text { then } \left.a(x, t)=\frac{a}{\sqrt{1-\alpha_{2}}} \quad \text { (for segment } T_{i i i}\right)
$$

Step 4: if $H(x, t)>H_{0}$ and $\frac{\partial H(x, t)}{\partial t}>0$

$$
\text { then } a(x, t)=\frac{a}{\sqrt{1+\alpha_{1}}}\left(\text { for segment } T_{i v}\right)
$$

\section{TRANSIENT ANALYSIS OF DIFFERENT PIPELINE MATERIALS CONFIGURATIONS USING WAVE DAMPING COEFFICIENT $(\alpha)$}

Transient analysis has been carried out for pipeline with different material and their two different combination configurations at three different flow velocities. It is evaluated that a sequential variation in the pressure wave velocity has damped transient pressure amplitude rapidly in the pipeline. The computer programmes based on MOC method are modified by 
incorporating different values of wave damping coefficients $(\alpha)$ and water hammer equation was solved using modified MOC approach and these numerical results are further validated with experimental results. The numerical transient analysis has been performed by adopting the four different values of wave damping coefficient $(\alpha)$ corresponding to each twelve experimental conditions at valve location as per Table 2 .

Numerical Results obtained for all material pipelines at flow velocity equal to $0.5 \mathrm{~m} / \mathrm{s}$ are plotted along with experimental results as shown in Fig. 4. It may be seen that, high damping in amplitude of pressure wave was observed for higher value of wave damping coefficient. Similarly, for each pipe configuration and flow velocity condition the results obtained from modified MOC method using four different wave damping coefficient were compared with experimental results.

\section{EXPERIMENTAL VALIDATION}

Experiments have been performed at three different flow velocities for different materials and their combined configurations and pressure data has been recorded at all five pressure sensors locations shown in Fig. 2. Further numerical transient analysis has been carried out similar to experiments by conventional MOC approach using MATLAB programme. Comparison of results obtained from each numerical case has been performed with corresponding experimental case. Fig. 5 shows the comparison of transient values obtained from numerical analysis with experiments for all pipeline material at downstream side under flow condition $V_{0}=0.5 \mathrm{~m} / \mathrm{s}$.

It was observed from Fig. 5 that conventional MOC approach predicts very low damping effect compared with experimental results for all pipeline material and configurations. Similar trend was also observed for other two flow velocities $(0.8$ and $1.0 \mathrm{~m} / \mathrm{s})$ and also for different 
locations. The peaks of transient pressure obtained from numerical analysis were further compared with experimental values and found out nearly equal in magnitude. The deviation in the damping pattern of transient pressure between the experimental and numerical simulation showed that there are some other parameters especially dissipative effect and losses occurring inside pipelines, are responsible for damping characteristics in the system. Thus, it may be concluded that the conventional water hammer equations are not precise to predict water hammer behavior in pipelines. For validation, parameters related to dissipative effect and losses should be incorporated in numerical analysis and conventional water hammer equations need to be modified.

\section{DETERMINATION OF OPTIMUM WAVE DAMPING COEFFICIENT $(\alpha)$ AND ESTIMATION OF QUANTIFICATION ERROR}

For validation of improved numerical MOC method with experimental results, deviation from experimental values to corresponding values in numerical method for all four wave damping coefficient $(\alpha)$ were quantified at different time instant (peak and valley of the wave pattern) as shown in Fig. 6. The error between the experimental and numerical graphs (for four different $\alpha$ ) has been evaluated at selected locations as shown in Fig. 6 and standard procedure has been adopted to quantify the error between them. Error $\beta_{1}, \beta_{2}, \beta_{3}, \beta_{4}$ have been estimated using the software and deviation between experimental and numerical values for each selected

locations. Further, sum square error $(S S E)$ has been calculated for each wave damping coefficient by adding the square error for different locations as per Eq. 10. 


$$
(\operatorname{SSE})_{x}=\sum_{1}^{14}\left(\beta_{x}\right)^{2}
$$

where $x=1,2,3,4$ corresponding to each four wave damping coefficient $(\alpha)$

To estimate the optimum value of $(\alpha)$ for each case, sum square error is plotted against four different $(\alpha)$ values. The Fig. 7 shows the root sum square error vs $\alpha$ for each numerical case and minimum root sum square error along with optimum $\alpha$ have been obtained from the graph which is tabulated in Table 2. It may be seen that for all configurations of pipeline the proposed approach ensures better agreement between the experimental and modified numerical approach. By taking into account the variable pressure wave velocity as well as the dissipative effect at the wave front, the attenuation of the amplitude of the head oscillations are better reproduced for all simulation cases. From Fig. 8, It has been observed that dissipative losses in GRP pipeline is more compared with other pipeline configuration as optimum value of wave damping coefficient

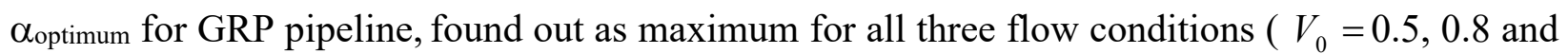
$1.0 \mathrm{~m} / \mathrm{s})$.

It is further observed that wave damping coefficient for MS pipeline is minimum compared with others configuration for all flow condition. Its means that wave damping in MS pipeline are less and wave damping effect is very slow compared with another pipeline configuration. Optimum value of $\alpha$ for GRP+MS pipeline configuration at all three flow condition, are found as very close to values obtained for GRP pipeline configuration 


\section{Conclusions}

There are several sources of energy dissipation in pipeline during water hammer events. The introduction and development of wave damping coefficient $(\alpha)$ in the water hammer equation significantly damped celerity in the pipeline and improved the wave damping by using water hammer equations to be in line with the experimental observations. The computer programmes for MOC method are improved with wave damping coefficient and results are experimentally validated. It is observed that the magnitude of $\alpha$ is 0.020 for MS pipeline material and 0.075 in GRP pipeline material and for combination configuration it is $0.015,0.065$ for MS+GRP and GRP+MS pipeline respectively for fluid velocity $0.5 \mathrm{~m} / \mathrm{s}$. For the fluid velocity $0.8 \mathrm{~m} / \mathrm{s}$, the value of $\alpha$ is observed as $0.015,0.085$ for MS and GRP pipeline material and $0.015,0.065$ for MS + GRP and GRP + MS pipeline respectively. For wave velocity $1 \mathrm{~m} / \mathrm{s}$, the value of $\alpha$ observed as 0.025 and 0.075 for MS and GRP pipeline and $0.020,0.070$ for GRP+MS, MS+GRP material pipeline respectively.

The smaller value of $\alpha$ is observed in metallic pipeline and higher in viscoelastic material. The wave damping coefficient is evaluated for all experimental cases. It can be concluded that the proposed approach by considering variable wave velocity in the analysis of water hammer gives better agreement between experimental and numerical results.

\section{Funding}

The first author expresses sincere thanks to Department of Hydro and Renewable Energy and Quality Improvement Program Centre, IIT, Roorkee, India for providing research facilities and to the All India Council for Technical Education (AICTE) Government of India for providing financial assistance in the form of research scholarship. 


\section{Ethics declarations}

\section{Conflict of Interest}

The authors declare that they have no conflict of interest.

\section{Consent to Participate}

The authors declare that they consent to participate.

\section{Consent to Publish}

The authors declare that they consent to publish.

Data Availability

Please see the attachment. 


\section{Notation}

\begin{tabular}{|c|c|}
\hline$A_{p}=$ & Cross- sectional pipe area \\
\hline$a=$ & Standard pressure wave velocity (celerity) \\
\hline$a_{c}=$ & Pressure wave velocity under compression \\
\hline$a_{d}=$ & Pressure wave velocity under decompression \\
\hline$D=$ & Inside diameter of the pipeline \\
\hline$E=$ & Young modulus of elasticity of pipeline material \\
\hline$T=$ & Thickness of pipeline wall \\
\hline$E_{k i n}=$ & Kinetic energy of the fluid \\
\hline$E_{f}=$ & Elastic energy of the fluid \\
\hline$E_{m}=$ & Elastic energy of the material \\
\hline$g=$ & Gravitational constant \\
\hline$H=$ & Piezometric head \\
\hline$\Delta H=$ & Change in pressure head \\
\hline$H_{0}=$ & Initial pressure head \\
\hline$K=$ & Bulk modulus of the water \\
\hline$L=$ & Length of the pipeline \\
\hline$\Delta p=$ & Change in pressure \\
\hline$Q=$ & Flow discharge \\
\hline$R=$ & Pipeline resistance coefficient \\
\hline$T=$ & Pipe thickness \\
\hline
\end{tabular}




$\begin{array}{ll}t_{C}= & \text { Valve closure time } \\ \Delta t= & \text { Time step } \\ V_{0} & \text { Mean flow velocity of the fluid } \\ \Delta x= & \text { Spatial step } \\ \varepsilon= & \text { Coughness height of the pipeline material } \\ \alpha= & \text { Coefficient of dissipation in compression phase } \\ \alpha_{1}= & \text { Coefficient of dissipation in decompression phase } \\ \alpha_{2}= & \end{array}$

\section{Abbreviation}

1D One dimensional abbreviation

2D Two dimensional abbreviation

DAQ Data Acquisition System

Eq. Equation

GRP Glass Reinforcement Fibre Plastic

HPP Hydropower Plant

MOC Method of Characteristics

MS Mild Steel

MATLAB Matrix Laboratory 


\section{References}

[1] C.C. Bonin, Water-Hammer Damage to Oigawa Power Station, J. Eng. Power. 82 (1960) 111. https://doi.org/10.1115/1.3672721.

[2] A. Bergant, A. Tijsseling, J. Vítkovský, D. Covas, A. Simpson, M. Lambert, E.I. Litostroj, Further Investigation of Parameters Affecting Water Hammer Wave Attenuation, Shape and Timing Part 1 : Mathematical Tools, J. Hydraul. Res. 46 (2008) 373-381.

[3] V. Yuzhanin, V. Popadko, T. Koturbash, V. Chernova, R. Barashkin, Predictive control and suppression of pressure surges in main oil pipelines with counter-running pressure waves, Int. J. Press. Vessel. Pip. 172 (2019) 42-47. https://doi.org/10.1016/j.ijpvp.2019.03.015.

[4] A. Adamkowski, M. Lewandowski, Preventing destructive effects of water hammer in hydropower plant penstocks, in: Hydropower Dev. Eur., 2015.

[5] A. Triki, M. Fersi, Further investigation on the water-hammer control branching strategy in pressurized steel-piping systems, Int. J. Press. Vessel. Pip. 165 (2018) 135-144. https://doi.org/10.1016/j.ijpvp.2018.06.002.

[6] R. Kumar, S.K. Singal, Penstock material selection in small hydropower plants using MADM methods, Renew. Sustain. Energy Rev. 52 (2015) 240-255. https://doi.org/10.1016/j.rser.2015.07.018.

[7] M. Kandil, A.M. Kamal, T.A. El-Sayed, Effect of pipematerials on water hammer, Int. J. Press. Vessel. Pip. 179 (2020) 1-8. https://doi.org/10.1016/j.ijpvp.2019.103996.

[8] Gong, Jinzhe, M.L. Stephenson, M.F. Lambart, A.C. Zecchin, A.R. Simpson, Pressure 
surge suppresion using a metallic-plastic-metallic pipe configuration, J. Hydraul. Eng. 144 (2018).

[9] M. Larson, L. Jonsson, Elastic Properties of Pipe Materials During Hydraulic Transients, J. Hydraul. Eng. 117 (1991) 1317-1331.

[10] E.B. Wylie, V.. Streeter, Fluid Transients, 1st Editio, Prentice Hall, New York, 1978.

[11] M.H. Chaudhry, Applied Hydraulic Transients, 3rd Editio, Springer-Verlag, New York, 2014. https://doi.org/10.1007/978-1-4614-8538-4.

[12] A. Bergant, A.S. Tijsseling, J.P. Vítkovský, D.I.C. Covas, A.R. Simpson, M.F. Lambert, Parameters Affecting Water-Hammer Wave Attenuation, Shape and Timing-Part 1:

Mathematical Tools, J. Hydraul. Res. 46 (2008) 373-381. https://doi.org/10.3826/jhr.2008.2848.

[13] D.J. Wood, Waterhammer Analysis-Essential and Easy (and Efficient), J. Environ. Eng. 131 (2005) 1123-1131. https://doi.org/10.1061/(ASCE)0733-9372(2005)131:8(1123).

[14] M. Mitosek, R. Szymkiewicz, Wave Damping and Smoothing in the Unsteady Pipe Flow, J. Hydraul. Eng. 138 (2012) 619-628. https://doi.org/10.1061/(ASCE)HY.19437900.0000571.

[15] A. Adamkowski, Analysis of Transient Flow in Pipes with Expanding or Contracting Sections, J. Fluids Eng. 125 (2003) 716. https://doi.org/10.1115/1.1593703.

[16] H.-F. Duan, M. Ghidaoui, P.J. Lee, Y.-K. Tung, Unsteady Friction and Visco-Elasticity in Pipe Fluid Transients, J. Hydraul. Res. 483 (2010) 22-1686. https://doi.org/10.1080/00221681003726247. 
[17] W. Zeilke, Frequency dependent friction in transient pipe flow, J. Basic Eng. 90 (1968) $109-115$.

[18] A.. Trikha, An efficient method or simulating frequency-dependent friction in transient liquid flow, J. Fluids Eng. 97 (1975) 97-105.

[19] A. Bergant, A.R. Simpson, A.S. Tijsseling, Water hammer with column separation: A historical review, J. Fluids Struct. 22 (2006) 135-171. https://doi.org/10.1016/j.jfluidstructs.2005.08.008.

[20] J. Izquierdo, P.. Iglesias, Mathematical Modelling of Hydraulic Transients in Simple Systems, Math. Comput. Model. 7177 (2002) 801-812.

[21] M. Mitosek, Study of transient vapour cavitation in series pipe systems, J .Hydraul .Eng.,. $126(2000) 904-911$.

[22] M. Mitosek, R. Szymkiewicz, Reservoir Influence on Pressure Wave Propagation in Steel Pipes, 142 (2016) 1-5. https://doi.org/10.1061/(ASCE)HY.1943-7900.0001140. 
Table 1: Characteristics of pipelines used

\begin{tabular}{|l|c|c|}
\hline parameter & MS Pipeline & GRP Pipeline \\
\hline pipeline length $(\mathrm{m})$ & 39 & 39 \\
\hline inside diameter of pipe $D(\mathrm{~m})$ & 0.08 & 0.08 \\
\hline pipeline wall thickness $T(\mathrm{~m})$ & 0.006 & 0.01 \\
\hline roughness $\varepsilon(\mathrm{m})$ & 0.00006 & 0.000003 \\
\hline pressure head $H_{0}(\mathrm{~m})$ & 80 & 80 \\
\hline flow velocities $V_{0}\left(\mathrm{~ms}^{-1}\right)$ & $0.5,0.8,1$ & $0.5,0.8,1$ \\
\hline pressure wave velocity $a\left(\mathrm{~ms}^{-1}\right)$ & 1350 & 900 \\
\hline
\end{tabular}


Table 2: Numerical cases with corresponding wave damping coefficient

\begin{tabular}{|c|c|c|c|c|c|}
\hline \multirow{3}{*}{ Pipe configuration } & \multirow{2}{*}{$\begin{array}{c}\text { Flow velocity } \\
(\mathbf{m} / \mathbf{s})\end{array}$} & \multicolumn{4}{|c|}{ Wave damping coefficient } \\
\cline { 2 - 6 } & & $\alpha_{1}$ & $\alpha_{2}$ & $\alpha_{3}$ & $\alpha_{4}$ \\
\hline \multirow{3}{*}{ MS Pipeline } & 0.5 & 0.010 & 0.015 & 0.020 & 0.025 \\
\cline { 2 - 6 } & 0.8 & 0.010 & 0.015 & 0.020 & 0.025 \\
\cline { 2 - 6 } & 1 & 0.005 & 0.010 & 0.015 & 0.020 \\
\hline \multirow{3}{*}{ GRP Pipeline } & 0.5 & 0.070 & 0.075 & 0.080 & 0.085 \\
\cline { 2 - 6 } & 0.8 & 0.075 & 0.080 & 0.085 & 0.090 \\
\cline { 2 - 6 } & 1 & 0.070 & 0.075 & 0.080 & 0.085 \\
\hline \multirow{3}{*}{ MS+GRP Pipeline } & 0.5 & 0.010 & 0.015 & 0.020 & 0.025 \\
\cline { 2 - 6 } & 0.8 & 0.010 & 0.015 & 0.020 & 0.025 \\
\cline { 2 - 6 } & 1 & 0.010 & 0.015 & 0.020 & 0.025 \\
\hline \multirow{3}{*}{ GRP+MS Pipeline } & 0.5 & 0.060 & 0.065 & 0.070 & 0.075 \\
\cline { 2 - 6 } & 0.8 & 0.060 & 0.065 & 0.070 & 0.075 \\
\cline { 2 - 6 } & 1 & 0.065 & 0.070 & 0.075 & 0.080 \\
\hline
\end{tabular}


Figures

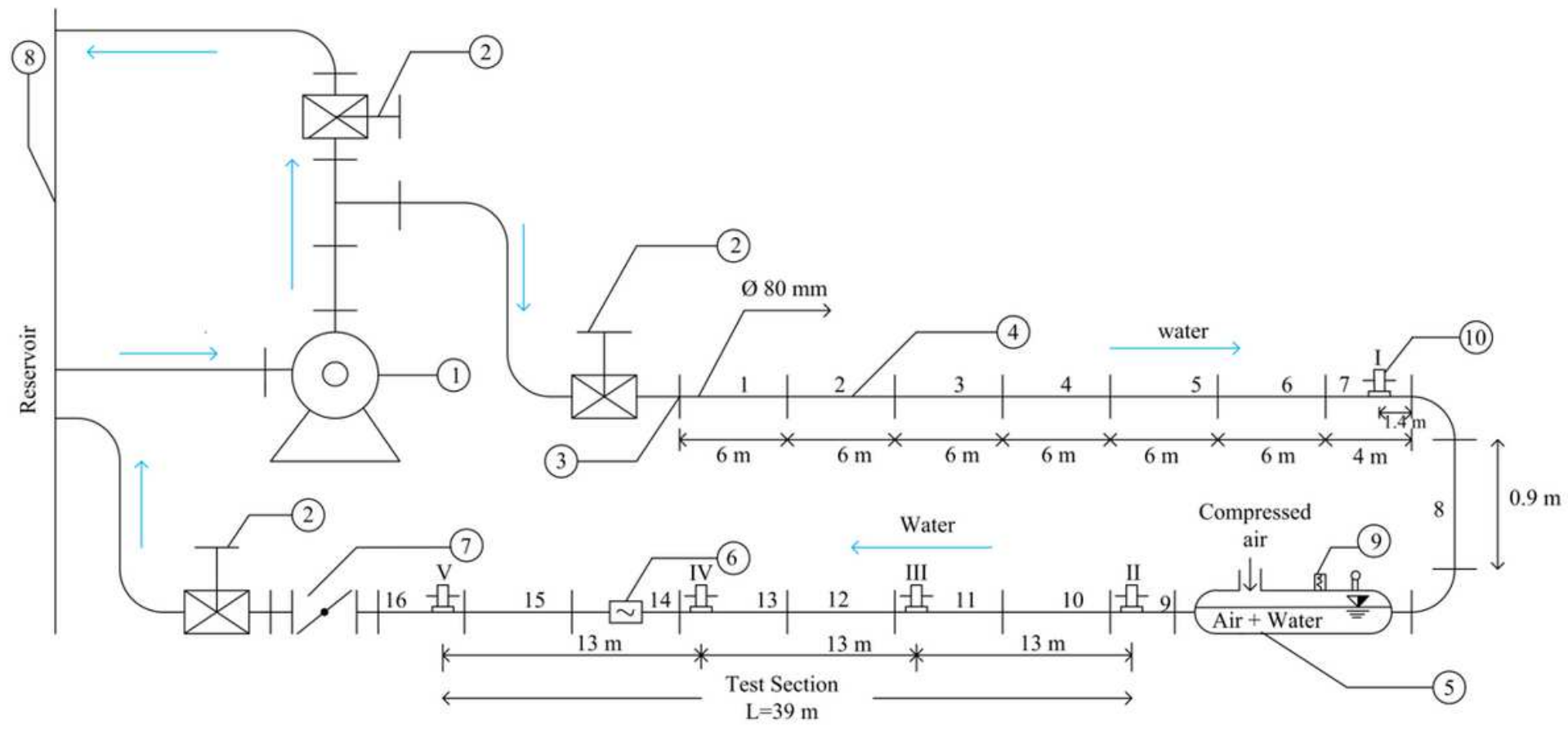

(1) Centrifugal Pump (2) Gate Valve (3) Flange (4) Pipe (5) Air Vessel

(6) Ultrasonic Flowmeter (7) Butterfly Valve (8) Reservoir (9) Safety valve (10) Pressure Sensor (I,II,III,IV and V)

Figure 1

Schematic diagram of water hammer experimental setup 


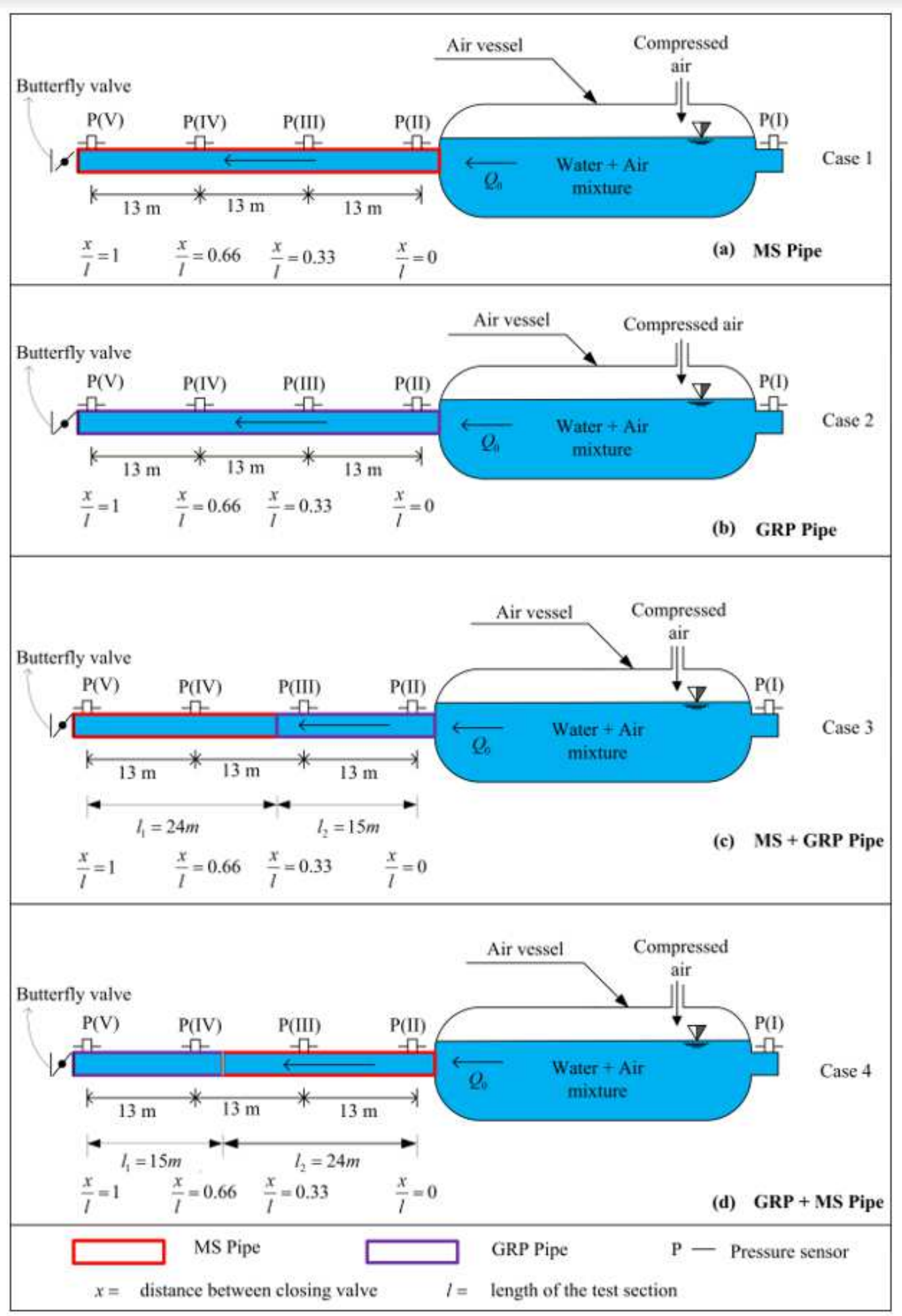

\section{Figure 2}

Experimental set up with different pipe material and combination in series 


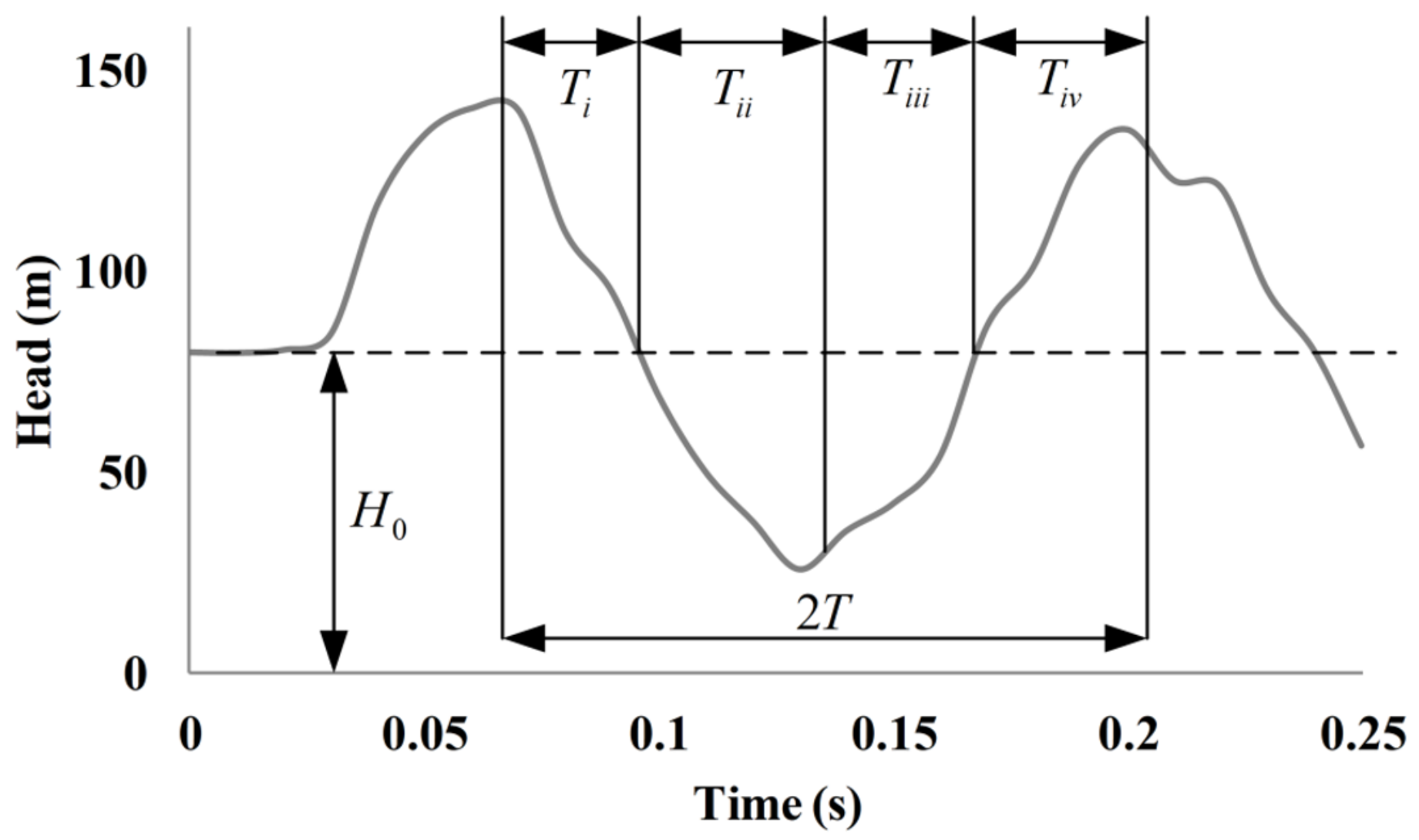

Figure 3

Head oscillation at valve of the MS pipeline 


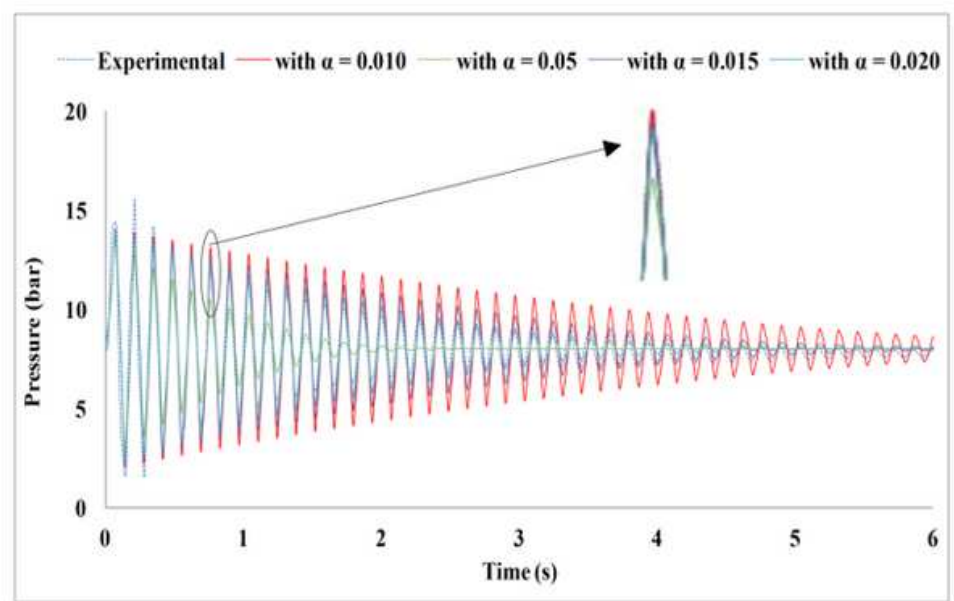

(a) MS Pipeline

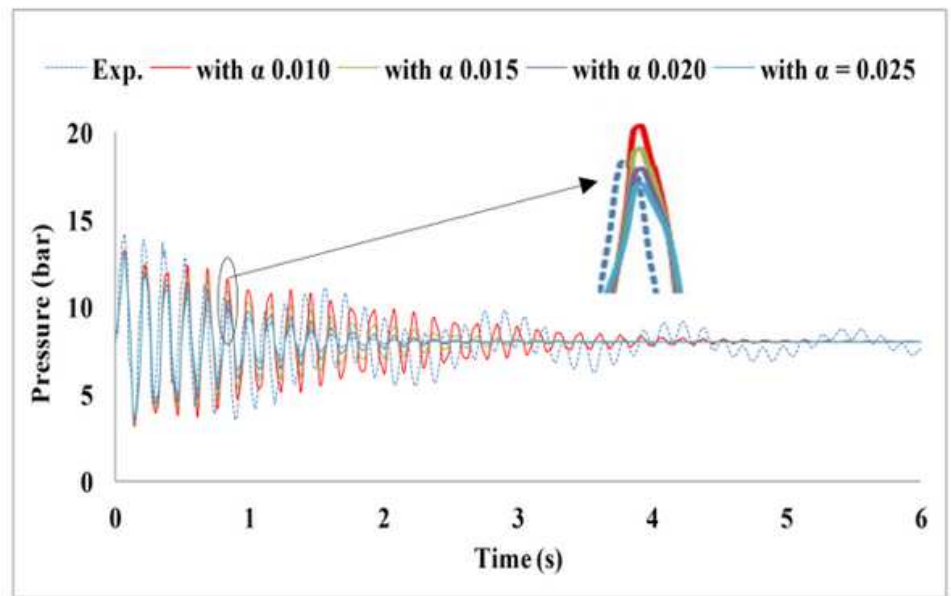

(c) MS+GRP Pipeline

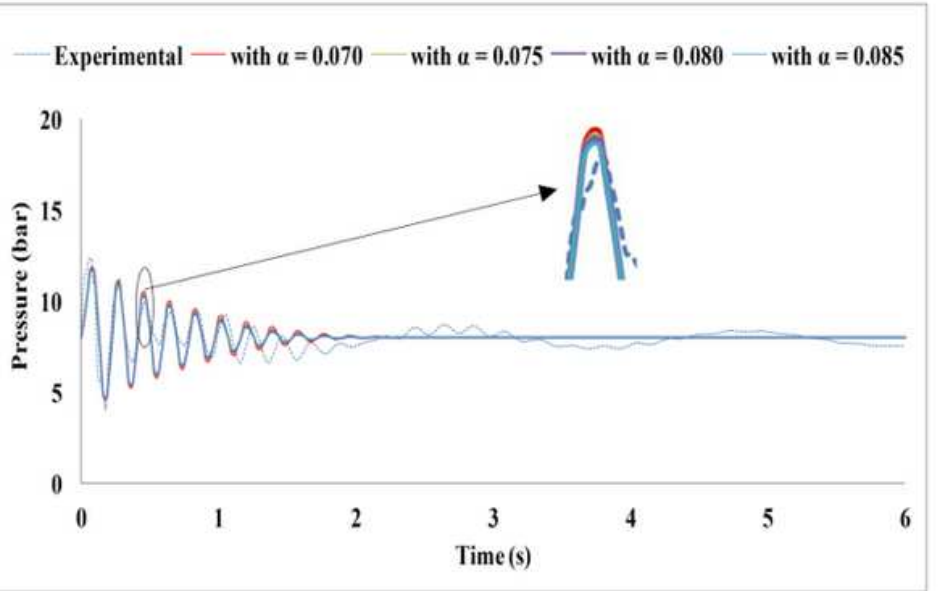

(b) GRP Pipeline

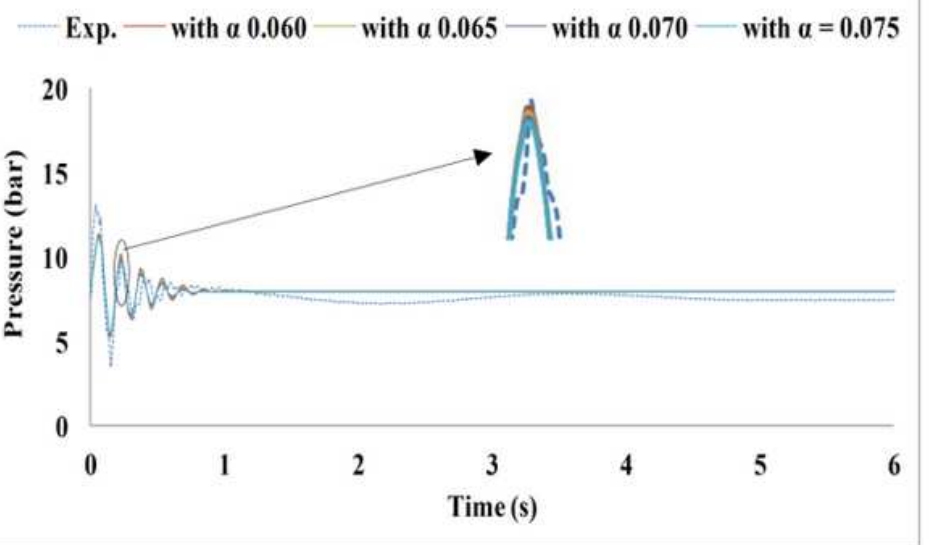

(d) GRP + MS Pipeline

\section{Figure 4}

Effect of different wave damping coefficient on amplitude of pressure wave 


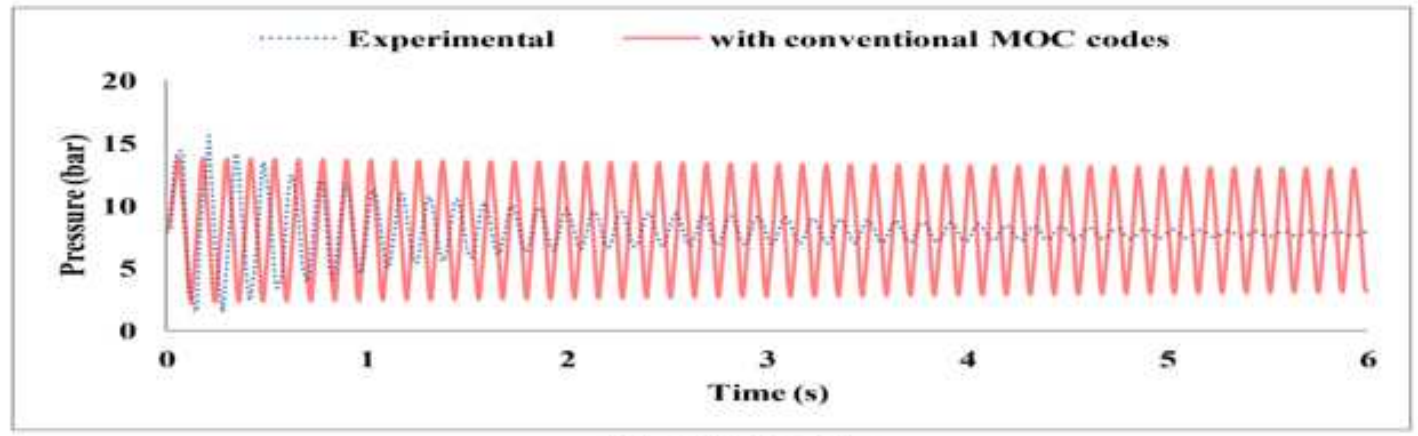

(a) MS Pipeline

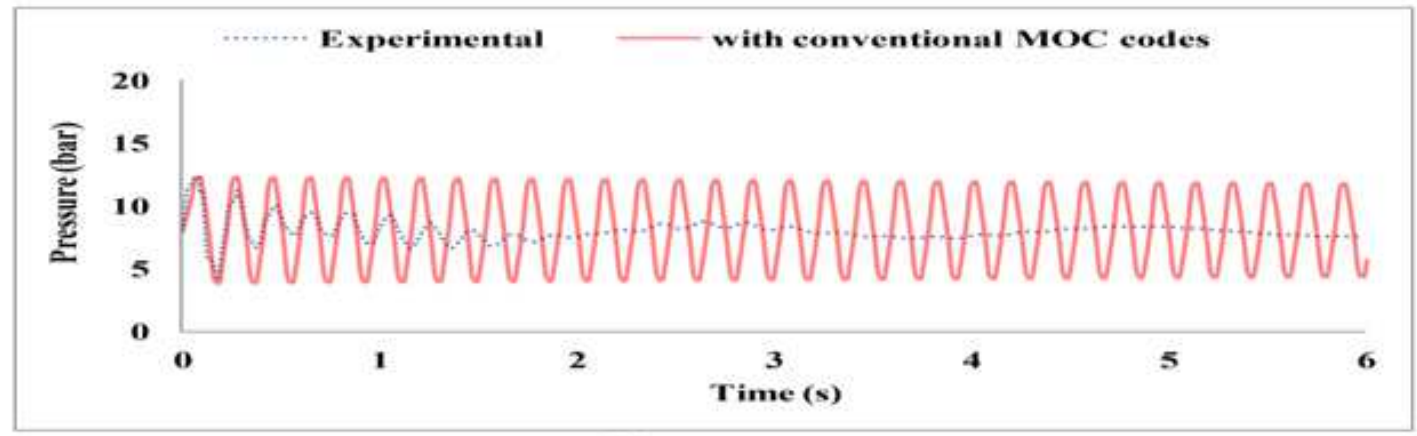

(b) GRP Pipeline

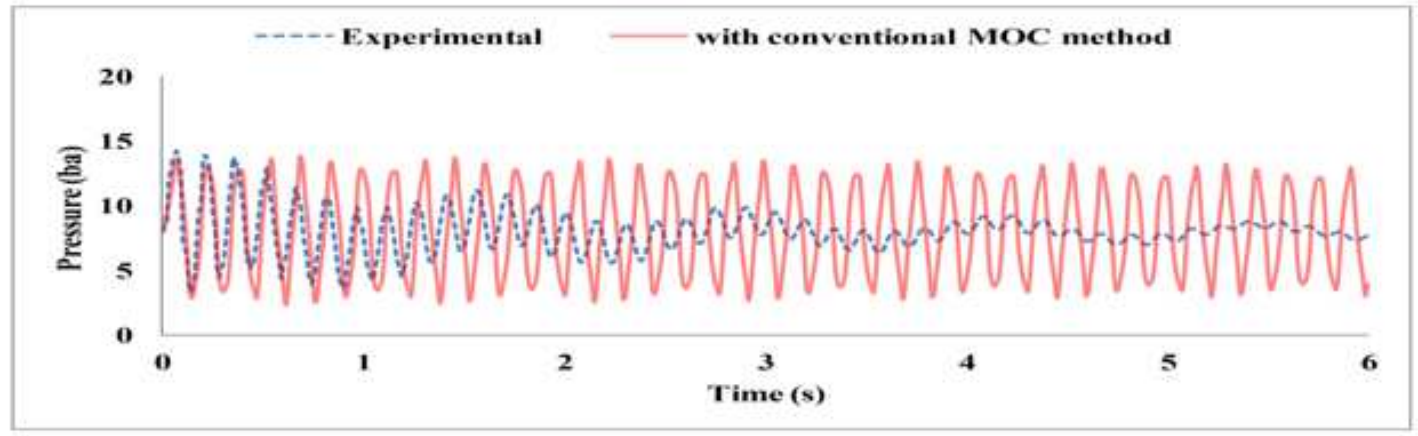

(c) MS+GRP Pipeline

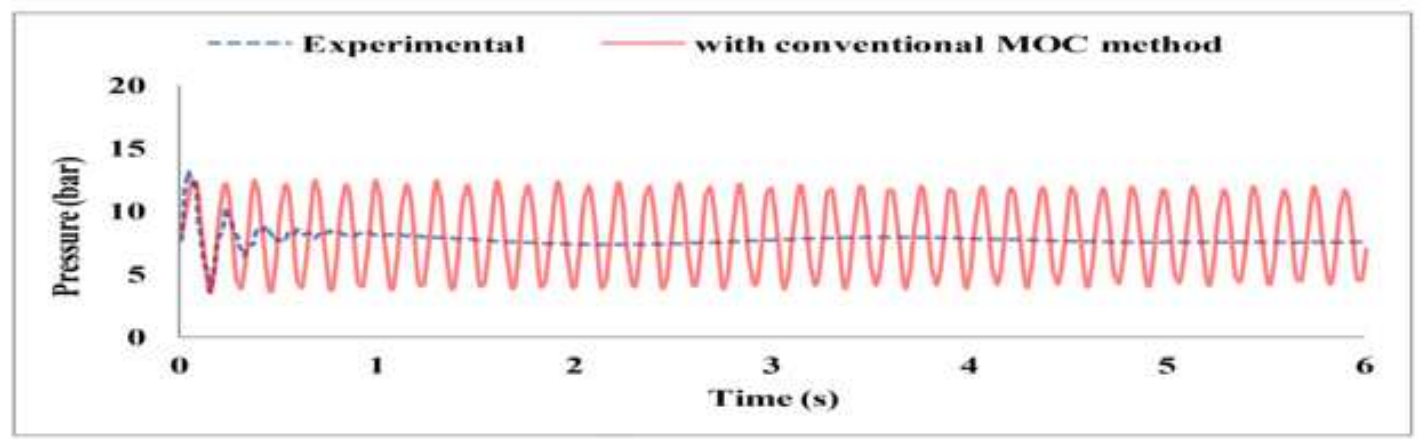

(d) GRP+MS Pipeline

Figure 5

Comparison of transient pressure for all different material configurations at $\mathrm{Vo}=0.5 \mathrm{~m} / \mathrm{s}$ 

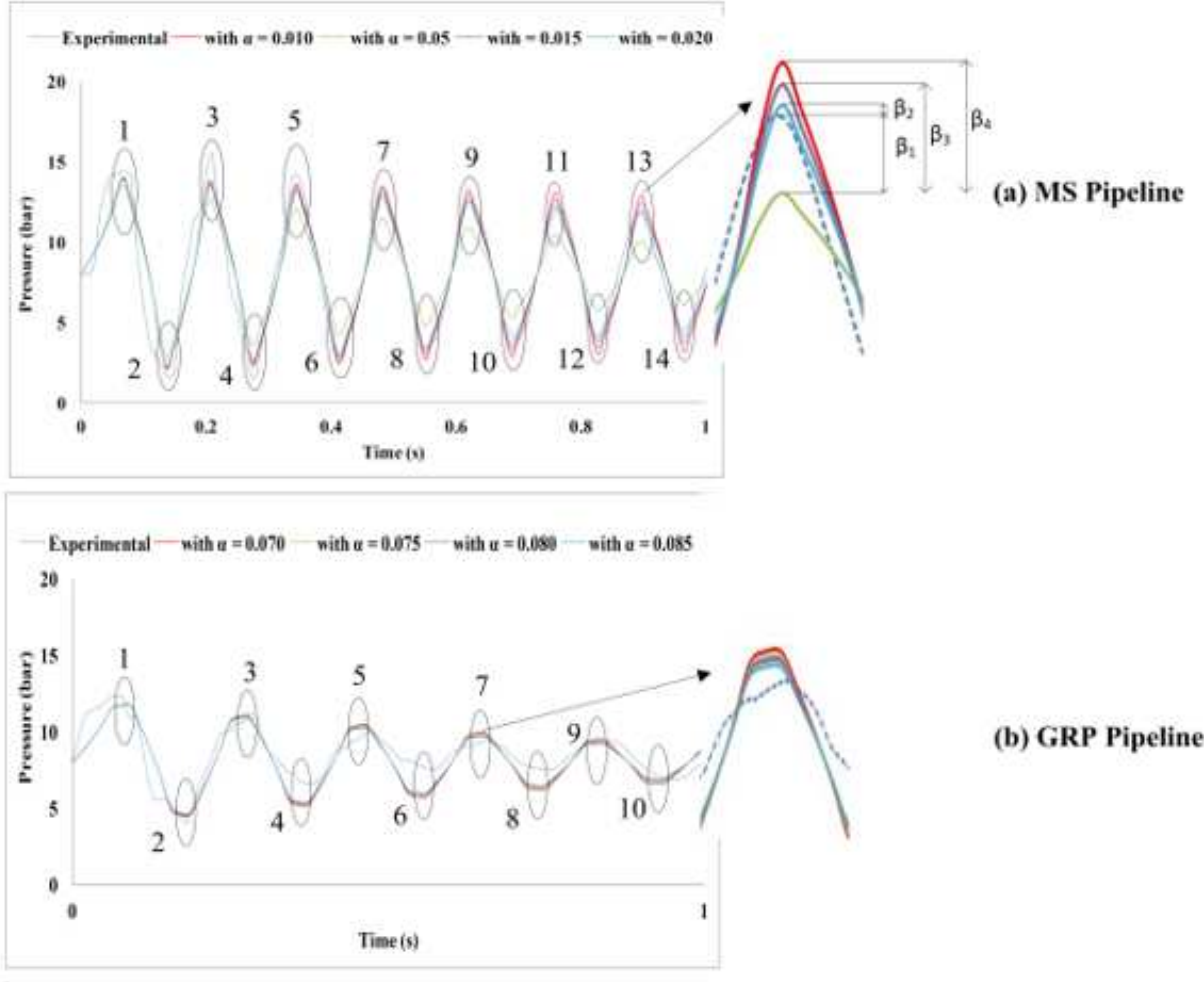

\section{(b) GRP Pipeline}

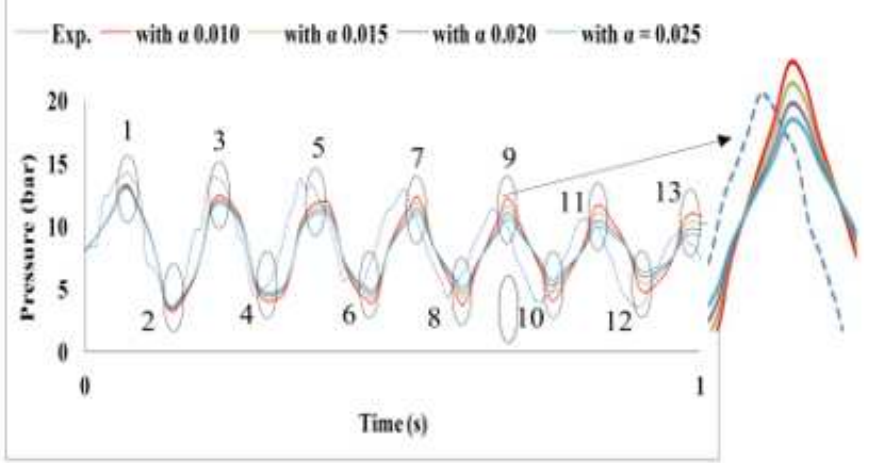

(c) MS + GRP Pipeline

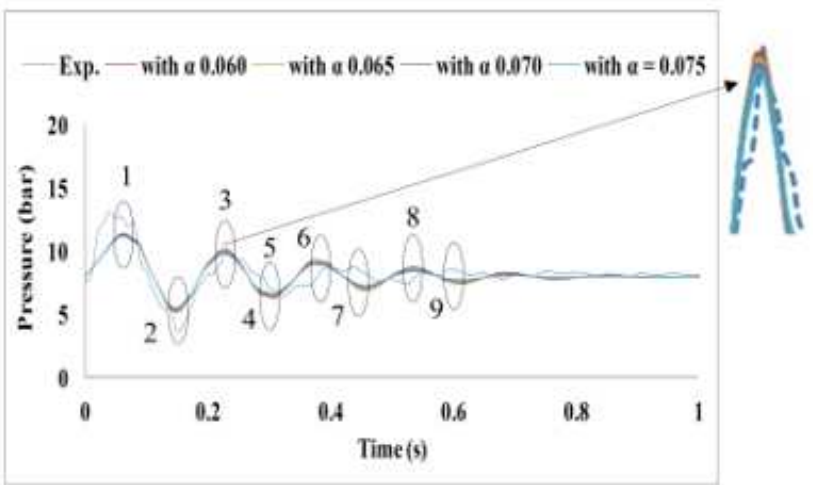

(d) GRP + MS Pipeline

\section{Figure 6}

Quantification of deviation from experimental results 


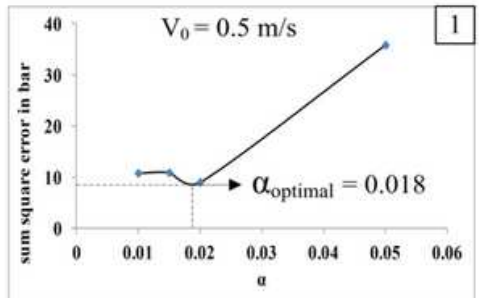

(a) MS Pipeline

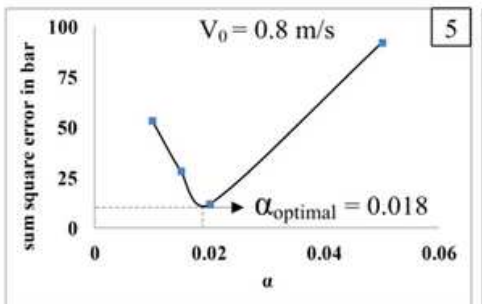

(a) MS Pipeline

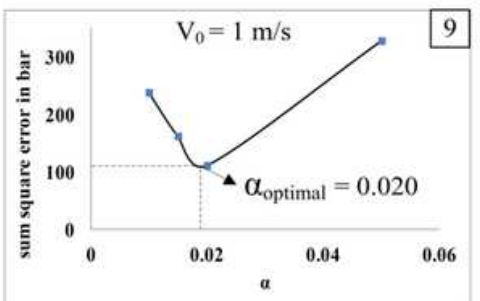

(a) MS Pipeline

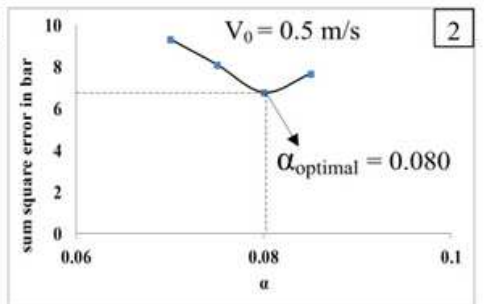

(b) GRP Pipeline

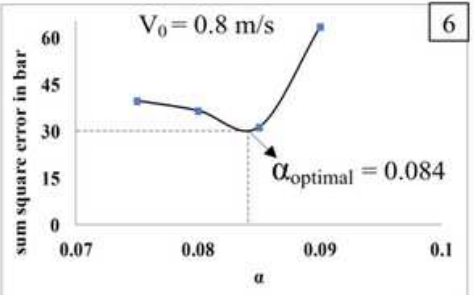

(b) GRP Pipeline

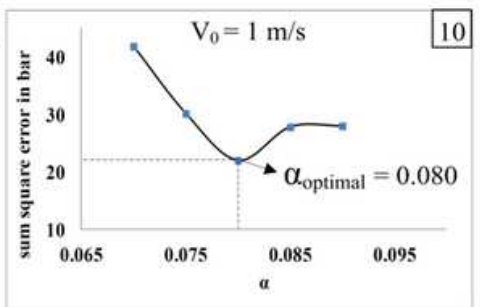

(b) GRP Pipeline

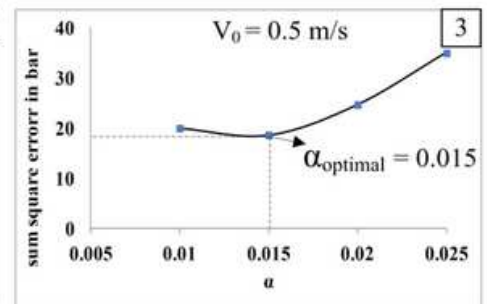

(c) MS+GRP Pipeline

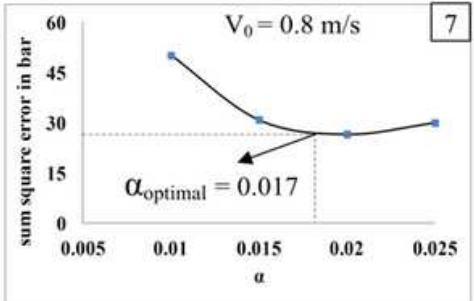

(c) MS+GRP Pipeline

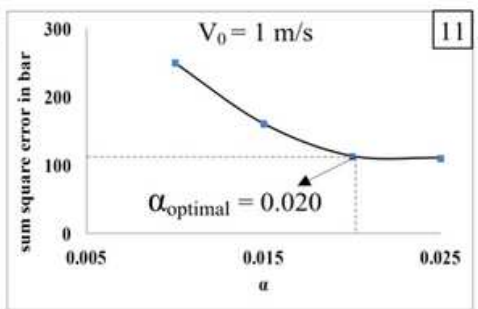

(c) MS+GRP Pipeline

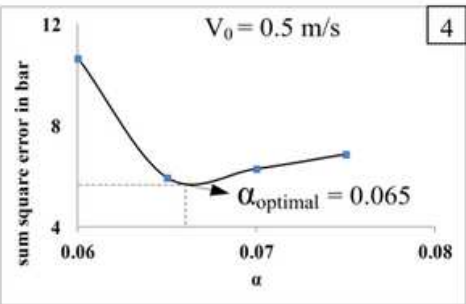

(d) GRP+MS Pipeline

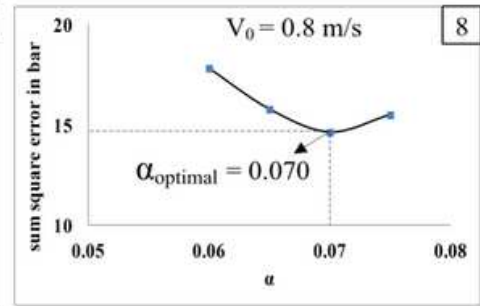

(d) GRP+MS Pipeline

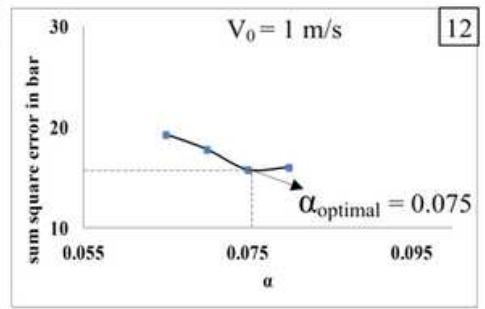

(d) GRP+MS Pipeline

Figure 7

Optimum value of a for each numerical case 


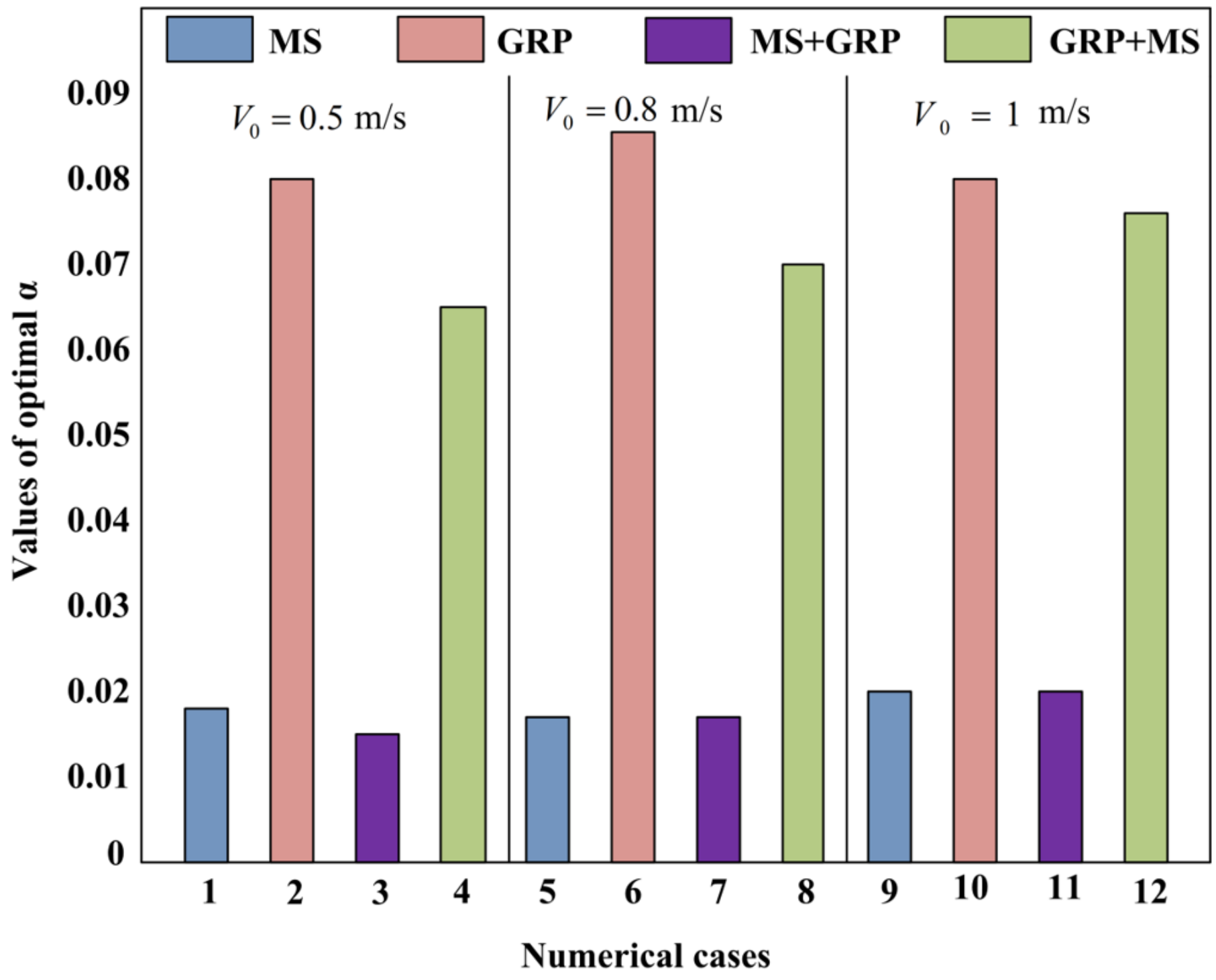

Figure 8

Values of optimum a obtained from each numerical case 